\title{
Spectroscopic observations of the rapid rotating post-AGB star IRAS 05381+1012 ${ }^{\star}$
}

\author{
C. B. Pereira and F. Roig \\ Observatório Nacional, Rua José Cristino 77, CEP 20921-400, São Cristóvão, Rio de Janeiro-RJ, Brazil \\ e-mail: claudio@on.br \\ Received 22 October 2005 / Accepted 15 January 2006 \\ ABSTRACT

\begin{abstract}
We report on the high-resolution stellar parameters and abundance analysis of the rapidly rotating post-AGB star IRAS $05381+1012$. Analysis of high-resolution spectra shows that IRAS $05381+1012$ has an effective temperature of $T_{\text {eff }}=5200 \pm 100 \mathrm{~K}$ and a surface gravity of $\log g=1.0 \pm 0.5$ corresponding to a spectral type G(2-3)I. These parameters result in an estimated luminosity of $970 L_{\odot}$ and a distance of $2700 \mathrm{pc}$. We also show that IRAS $05381+1012$ has a projected rotational velocity $v \sin i=40 \pm 10 \mathrm{~km} \mathrm{~s}{ }^{-1}$. The abundance analysis based on a few available lines reveals that this star is an iron-deficient object with $[\mathrm{Fe} / \mathrm{H}]=-0.8$. We also analyze the abundance pattern and compare it to other classes of stars with similar stellar parameters.
\end{abstract}

Key words. stars: AGB and post-AGB - stars: abundances

\section{Introduction}

Abundance determination in post-AGB stars is an important tool for constraining the evolutionary models of low/intermediate main-sequence stars (masses $M_{\star} \leq 8 M_{\odot}$ ) in the last stages of their evolution. The photospheric chemical composition of post-AGB stars, which has been widely investigated during the last years, revealed a rather complex picture; see van Winckel (2003) for the most recent review. These stars can be divided basically into four categories: (i) the hot post-AGB stars, where some are carbon deficient (McCausland et al. 1992) while others, such as LSIV $-12^{\circ} 111$ and HD 341617, are not (Ryans et al. 2003); (ii) stars that present signatures of s-process enrichment in their optical spectra, which can also be referred to as " $21 \mu \mathrm{m}$ stars" due to presence of a C-rich circumstellar feature at $21 \mu \mathrm{m}$; (iii) stars that exhibit strong iron depletion, such as $[\mathrm{Fe} / \mathrm{H}]=-4.9$ for HR 4049 (Waelkens et al. 1991), and are also known as "metal-poor binary post-AGB stars"; and (iv) stars that are O-rich with a double-peaked SED and not enriched in s-process elements, such as HR 4912 (Luck et al. 1990).

However, not yet reported in the literature is a cold postAGB star with $G$ spectral type and a rapid rotational velocity. Here we present the abundance analysis of IRAS $05381+1012$, a post-AGB star candidate that will be shown to have rapid rotation. There are several references in the literature claiming that IRAS $05381+1012$ may be a post-AGB star: García-Lario et al. (1997), Hrivnak et al. (1994), Kwok (1993), and Fujii et al. (2002). Indeed, we show in the next sections that IRAS $05381+1012$ presents several characteristics of a post-AGB star.

We carried out high-resolution optical spectroscopy to derive the stellar parameters, photospheric abundances, and rotational velocity. Post-AGB stars and planetary nebulae exhibit a variety

* Based on observations made with the $1.52 \mathrm{~m}$ telescope at the European Southern Observatory (La Silla, Chile) under agreement with the CNPq-Observatório Nacional (Brazil). of shapes and several classification schemes have been already proposed, ranging from spherical through elliptical to bipolar (Meixner et al. 1999; Balick 1987). Among the different models developed to reproduce the observed morphologies, bipolar planetary nebulae shapes can result from the dust-driven winds of rotating AGB stars (Herwig 2004). Rotation should be responsible for the observed inhomogeneities, which would result from the interaction of the fast, ionized stellar wind with a dense equatorial circumstellar AGB envelope (Kwok 1982; Balick 1987).

This paper also shows how important it is to obtain highresolution spectra with a high $\mathrm{S} / \mathrm{N}$ ratio in order to analyze objects with F-G spectral type having rapid rotation. Since the number of absorption lines starts to increase as we move to later spectral types, and the lines become broader due to the fast rotation, they merge into each other and make it difficult to analyze the spectrum if the $\mathrm{S} / \mathrm{N}$ is poor.

\section{Observations}

The high-resolution spectra of IRAS $05381+1012$ analyzed in this work were obtained with the FEROS (Fiberfed Extended Range Optical Spectrograph) echelle spectrograph (Kaufer et al. 1999) of the $1.52 \mathrm{~m}$ ESO telescope at La Silla (Chile), on March 5th and December 12th, 1999. The FEROS spectral resolving power is $R=48000$, corresponding to 2.2 pixel of $15 \mu \mathrm{m}$, and the wavelength coverage goes from $4000 \AA$ to $9200 \AA$. The nominal $\mathrm{S} / \mathrm{N}$ ratio was evaluated by measuring the rms flux fluctuation in selected continuum windows, and the typical values were S/N 100-150 after $4500 \mathrm{~s}$ and $2 \times 3600 \mathrm{~s}$ of integration time, respectively. The spectra were reduced with the MIDAS pipeline reduction package consisting of the following standard steps: CCD bias correction, flat-fielding, spectrum extraction, wavelength calibration, correction of barycentric velocity, and spectrum rectification.

The low-resolution spectrum was obtained with the Boller \& Chivens spectrograph at the Cassegrain focus of the $1.52 \mathrm{~m}$ 


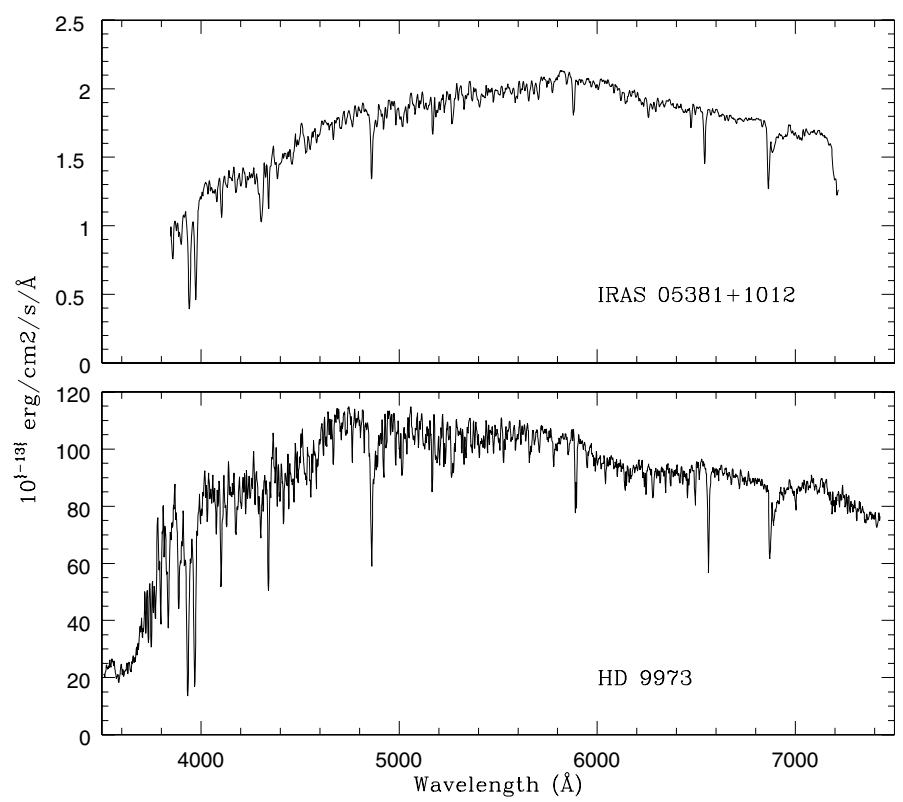

Fig. 1. Low-resolution spectra of IRAS 05381+1012 (top) and HD 9973 (bottom). The latter is an F5Iab star whose spectrum has been reddened by $E(B-V)=0.5$ for comparison. Note the very strong Ca II absorption lines appearing in both spectra at about $3900 \AA$.

ESO telescope at La Silla on March 3rd, 1999. A UV-flooded, thinned Loral Lesser CCD \#39 $(2048 \times 2048,15 \mu \mathrm{m} / \mathrm{pixel})$ was used as a detector, giving a high quantum efficiency in the blue and UV range. The instrument setup was the same used for a survey of post-AGB candidates carried out by Pereira \& Machado (2003) with the $1.52 \mathrm{~m}$ ESO telescope. We used the grating \#23, with $600 \mathrm{l} / \mathrm{mm}$, providing a resolution of $4.6 \AA$ and a range of $\lambda \lambda 3800-7500 \AA$.

The low resolution spectrum was reduced using standard IRAF tasks, from bias subtraction and flat-field correction through spectral extraction and wavelength and flux calibration. For this last task, the spectrophotometric standards LTT 3218, LTT 4816, and H600 from Hamuy et al. (1994) were also observed. Figure 1 (top panel) shows the reduced spectrum and compares it to the spectrum of a typical F5Iab star (HD 9973 from Jacoby et al. 1984), reddened by $E(B-V)=0.5$ (bottom panel). We can see that IRAS $05381+1012$ presents the very strong $\mathrm{Ca}$ II lines $\mathrm{H}$ and $\mathrm{K}$ in absorption, which are typical of F-type stars (Jacoby et al. 1984).

\section{Analysis and results}

\subsection{Stellar parameters and projected rotational velocities}

The spectrum of IRAS 05381+1012 exhibits absorption lines broadened by a very large rotational velocity. This is clearly shown in Fig. 2, where we compare a portion of the spectrum of IRAS $05381+1012$ (gray curve in top panel) with the spectra of the $\mathrm{D}^{\prime}$-type symbiotic star AS 201 (middle) and the post-AGB star IRAS $19386+0155$ (bottom). These two latter are characterized by their moderately large rotational velocity (Pereira et al. 2005) and a low rotational velocity (Pereira et al. 2004), respectively. In order to derive chemical abundances and to obtain spectroscopic estimates of the stellar parameters of IRAS $05381+1012$, we adopted spectrum synthesis techniques specific to objects with projected rotational velocities larger than about $20 \mathrm{~km} \mathrm{~s}^{-1}$. In particular, we followed the same approach used in the study of the $\mathrm{D}^{\prime}$-type rapid rotating symbiotic stars S190, AS 201, and HD 330036 (Smith et al. 2001; Pereira et al. 2005).

Due to the rapid rotation, the lines become blended, thereby preventing a photospheric analysis based on traditional methods that consist of measuring the individual lines' equivalent widths. Thus, it is necessary to carefully select those regions of the spectrum that contain broadened features whose respective strengths are approximately dominated by single species, such as Fe I, Fe II, Ca I, Ba II, and Mn I. For the present analysis, the chosen regions were in the intervals 6015-6030 $\AA$ (Fe I and Mn I lines), 6133-6152 A (Fe I, Fe II, Ba II and Si I lines), and 6436-6468 (Ca I lines). In order to obtain the best possible spectroscopic constraints on effective temperature and gravity, the ionization equilibrium requires finding not only isolated Fe I lines but also Fe II lines. Unfortunately, isolated Fe II lines are not very abundant in stellar spectra at the effective temperatures and gravities in cool stars, making the situation even worse due to the rapid rotation. In this study, we focused on analyzing a few good Fe I and Fe II features to fit the stellar parameters, as well as some $\mathrm{Ca}$ I and Mn I features to determine abundances and a Ba II feature as a monitor of s-process nucleosynthesis.

In order to determine the atmospheric parameters of the star, we produced synthetic spectra using the MOOG LTE synthesis code (Sneden 1973), together with a Kurucz \& Bell (1995) linelist and several Kurucz ATLAS9 model atmospheres. We computed a grid of synthetic spectra covering a range in effective temperature $\left(T_{\text {eff }}\right)$ from 4500 to $7000 \mathrm{~K}$, in surface gravity $(\log g)$ from 0.0 to 4.0 (with $g$ measured in $\mathrm{cm} \mathrm{s}^{-2}$ ), in microturbulence velocities $(\xi)$ from 1.0 to $4.0 \mathrm{~km} \mathrm{~s}^{-1}$, and five values of metallicity $[\mathrm{A} / \mathrm{H}]: 0.0,-0.5,-1.0,-1.5$, and -2.0 . Each of these synthetic spectra was compared to the observed spectra in the interval $6133-6152 \AA$, where there are relatively unblended Fe I and Fe II lines. More precisely, our determination of the atmospheric parameters rely on the fit of two Fe I lines at $6136.62 \AA$ and $6137.69 \AA$ and of two Fe II lines at $6147.74 \AA$ and $6149.26 \AA$, respectively. It is worth noting that these behave as "single" spectral lines, since the whole absorption profile is dominated by them.

The fit procedure can be summarized as follows: For each synthetic spectrum, corresponding to given values of $T_{\text {eff }}, \log g$, $\xi,[\mathrm{A} / \mathrm{H}]$, we first determined the abundance of $\mathrm{Fe} I$ and the projected rotational velocity $(v \sin i)$ that best reproduce the observed profile of the two Fe I lines mentioned above. This is done by searching for those values of Fe I abundance and of $v \sin i$ that minimize the merit function $\chi^{2}=\sum\left(f_{\text {synthetic }}-f_{\text {observed }}\right)^{2}(f$ represents the normalized spectral flux around the selected lines). Then, we repeated the procedure to determine the abundance of Fe II and the value of $v \sin i$ that reproduce the profile of the two Fe II lines mentioned above. In this way, we ended up with best-fit values of Fe I and Fe II abundances, and of $v \sin i$, for each set $T_{\text {eff }}, \log g, \xi,[\mathrm{A} / \mathrm{H}]$. The atmospheric parameters are then determined as those values of $T_{\text {eff }}, \log g, \xi$, and $[\mathrm{A} / \mathrm{H}]$ for which the abundance of Fe I equals the abundance of Fe II at the same projected rotational velocity. For IRAS $05381+1012$ the atmospheric parameters and rotational velocity determined in this way are: $T_{\text {eff }}=5200 \mathrm{~K}, \log g=1.0\left(\right.$ in $\left.\mathrm{cm} \mathrm{s}^{-2}\right), \xi=2.0 \mathrm{~km} \mathrm{~s}^{-1}$, $[\mathrm{A} / \mathrm{H}]=-1.5$, and $v \sin i=40 \mathrm{~km} \mathrm{~s}^{-1}$. The estimated $1 \sigma$ uncertainties in the atmospheric parameters are related to the resolution of the grid of synthetic spectra used, and indicate that $T_{\text {eff }}$ and $\log g$ are constrained to about $\pm 100 \mathrm{~K}$ and \pm 0.5 , respectively. The uncertainty in rotational velocity comes from the fit procedure and is about $\pm 10 \mathrm{~km} \mathrm{~s}^{-1}$. The derived values of $T_{\mathrm{eff}}$ 


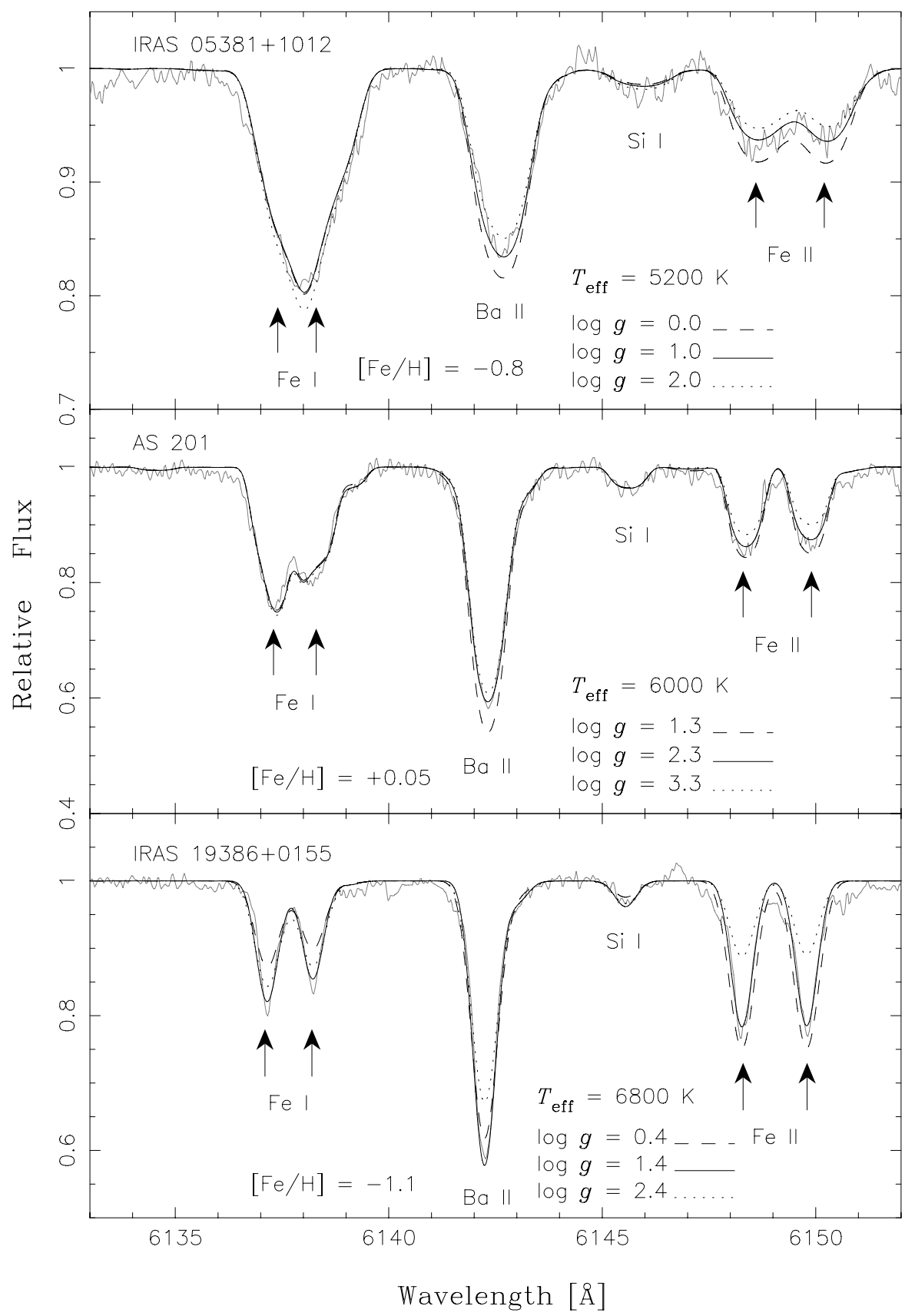

Fig. 2. Observed and synthetic spectra of IRAS 05381+1012 (top) with several lines showing rotationally broadened profiles. The observed spectrum (gray line) is compared to three synthetic spectra computed for three different surface gravities (dashed, full, and dotted lines). The full line is the best-fit spectrum. It is worth noting the sensitivity of the Fe II and Ba II lines strength to the different values of log $g$. The results are compared to similar results for the fast rotating D'-type symbiotic star AS 201 (middle) and the slow rotating post-AGB star IRAS 19386+0155 (bottom), both having slower rotational velocities than IRAS 05381+1012.

and $\log g$ suggest a G(2-3)I spectral type (Schmidt-Kaler 1982) that can be compared to the GI type given by Hrivnak et al. (1994).

Figure 2 (top) shows the observed spectrum of IRAS $05381+1012$ in the interval 6133-6152 $\AA$, together with three synthetic spectra: the one corresponding to the bestestimate atmospheric parameters for this star derived above, and two other synthetic spectra computed for the same atmospheric parameters except for $\log g$, which was varied by \pm 1.0 from its best estimate. It is interesting to note that smaller gravities tend to increase the lines' strength while larger gravities produce the contrary effect. This is particularly notorious in the Fe II lines and helps to validate our best estimate of $\log g=1.0$. This result is compared to similar data for the $\mathrm{D}^{\prime}$-type symbiotic star AS 201 and the post-AGB star IRAS 19386+0155, both having lower projected rotational velocities. In the case of AS 201 the estimated $v \sin i$ is $25 \pm 5 \mathrm{~km} \mathrm{~s}^{-1}$, while it is $8 \pm 1 \mathrm{~km} \mathrm{~s}^{-1}$ for IRAS $19386+0155$. These values may be compared to the $40 \pm 10 \mathrm{~km} \mathrm{~s}^{-1}$ estimated here for IRAS $05381+1012$, clearly indicating that the broader features in its spectrum are related to very rapid rotation.

In Fig. 3, the abundances (relative to the Sun) of Fe I and Fe II for IRAS $05381+1012$, as determined by our fit procedure, are shown in dependence of $T_{\text {eff }}$. In this plot, the values of $\log g, \xi$, 


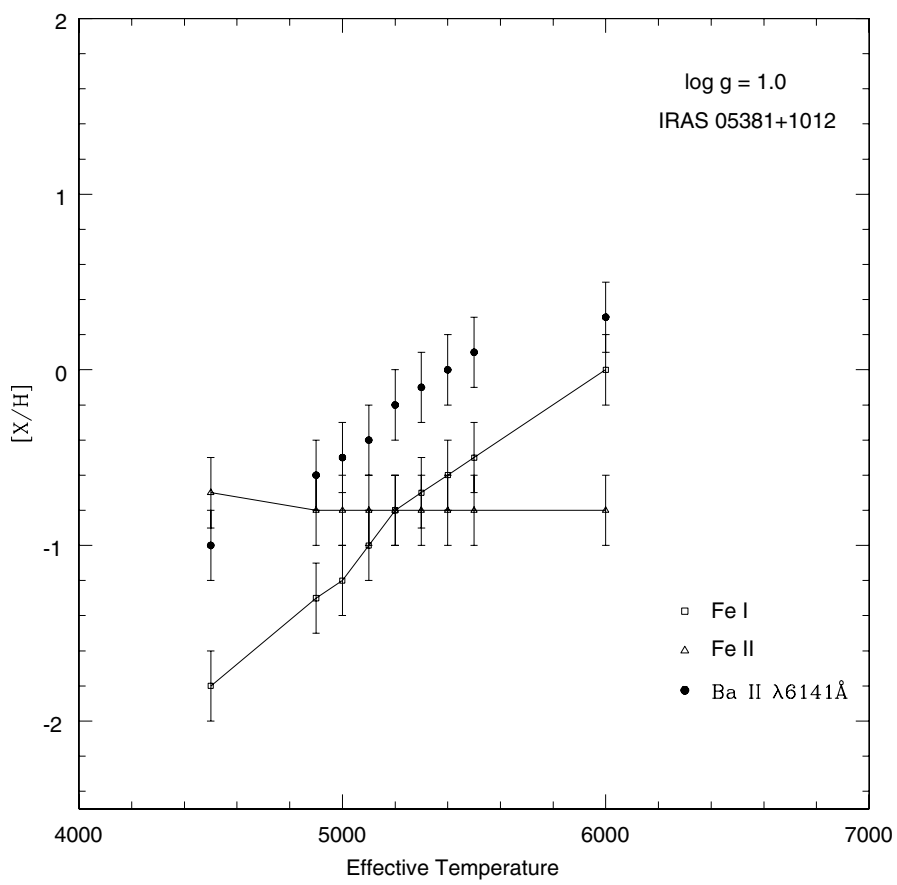

Fig. 3. Abundances $[\mathrm{Fe} I / H],[\mathrm{Fe} I \mathrm{I} / \mathrm{H}]$, and $[\mathrm{BaII} / \mathrm{H}]$ versus effective temperature, for different model atmospheres of IRAS 05381+1012, all having $\log g=1.0$ and $\xi=2.0 \mathrm{~km} \mathrm{~s}^{-1}$. Fe I and Fe II give the same abundances at $T_{\text {eff }}=5200 \mathrm{~K}$.

and $[\mathrm{A} / \mathrm{H}]$ are kept fixed and correspond to the best estimates for this star. Actually, Fig. 3 is a partial representation of our method for determining the atmospheric parameters. We choose this representation because the abundances of Fe I and Fe II are much more sensitive to changes in $T_{\text {eff }}$ than to changes in the other parameters. We note that the Fe I and Fe II abundances intercept each other at $T_{\text {eff }}=5200 \mathrm{~K}$, which is precisely the best estimate for effective temperature. From Fig. 3 it is possible to derive the actual abundance of Fe I in IRAS $05381+1012$, which is $6.72 \pm 0.2$. This figure also shows the abundances of Ba II derived from the $6142 \AA$ line (cf. Fig. 2). The actual abundance of this element is the one obtained at $T_{\text {eff }}=5200 \mathrm{~K}$, that is $1.93 \pm 0.2$.

\subsection{Abundance analysis}

The next step was to determine abundances via spectral synthesis for a sample of chemical elements of interest. The high, projected rotational velocity is a limiting factor in determining abundances from weak lines that become washed away in rotationally broadened profiles. We derived abundances of Fe, $\mathrm{Ba}, \mathrm{Ca}, \mathrm{Mg}, \mathrm{Si}, \mathrm{Ni}$, and $\mathrm{Mn}$. In Table 1, we list all the studied elements and atomic transitions, together with their respective atomic parameters (excitation potentials and $g f$-values).

Figure 4 shows the observed spectra in the regions around $6020 \AA$ and around $6440 \AA$, where several Fe I and $\mathrm{Ca}$ I lines are observed, respectively. This figure also shows the best-fit synthetic spectra for these two regions. The abundance of Mn I was based on the best fit of the $\lambda 6021$ line (bottom panel), and the hyperfine-structure components of this line were explicitly considered in the calculations (the $g f$-values for Mn I are from Booth et al. 1983).

Table 2 gives the abundance results for IRAS $05381+1012$, indicating that this star is slightly underabundant compared to the Sun in all the $\alpha$-elements studied. It is clearly an irondeficient star, although its abundance pattern is basically solar
Table 1. Absorption lines studied in IRAS 05381+1012.

\begin{tabular}{ccccc}
\hline \hline$\lambda(\AA)$ & Species & $\chi(\mathrm{eV})$ & \multicolumn{1}{c}{$g f$} & Ref. \\
\hline 8736.040 & Mg I & 5.94 & $4.571 \mathrm{e}-01$ & WSM \\
6145.020 & Si I & 5.61 & $3.311 \mathrm{e}-02$ & T90 \\
6439.075 & Ca I & 2.52 & $1.176 \mathrm{e}+00$ & LB85 \\
6449.808 & Ca I & 2.52 & $2.820 \mathrm{e}-01$ & S86 \\
6455.605 & Ca I & 2.52 & $3.502 \mathrm{e}-02$ & LB85 \\
6462.567 & Ca I & 2.52 & $0.073 \mathrm{e}+01$ & T90 \\
6021.761 & Mn I & 3.07 & $4.721 \mathrm{e}-02$ & BSW \\
6767.770 & Ni I & 1.83 & $6.761 \mathrm{e}-03$ & MFW \\
6141.727 & Ba II & 0.70 & $1.862 \mathrm{e}+00$ & T90 \\
\hline
\end{tabular}

BSW: Booth et al. (1983); LB85: Luck \& Bond (1985); MFW: Martin et al. (1988); S86: Smith et al. (1986); T90: Thévenin (1990); WSM: Wiese et al. (1969).
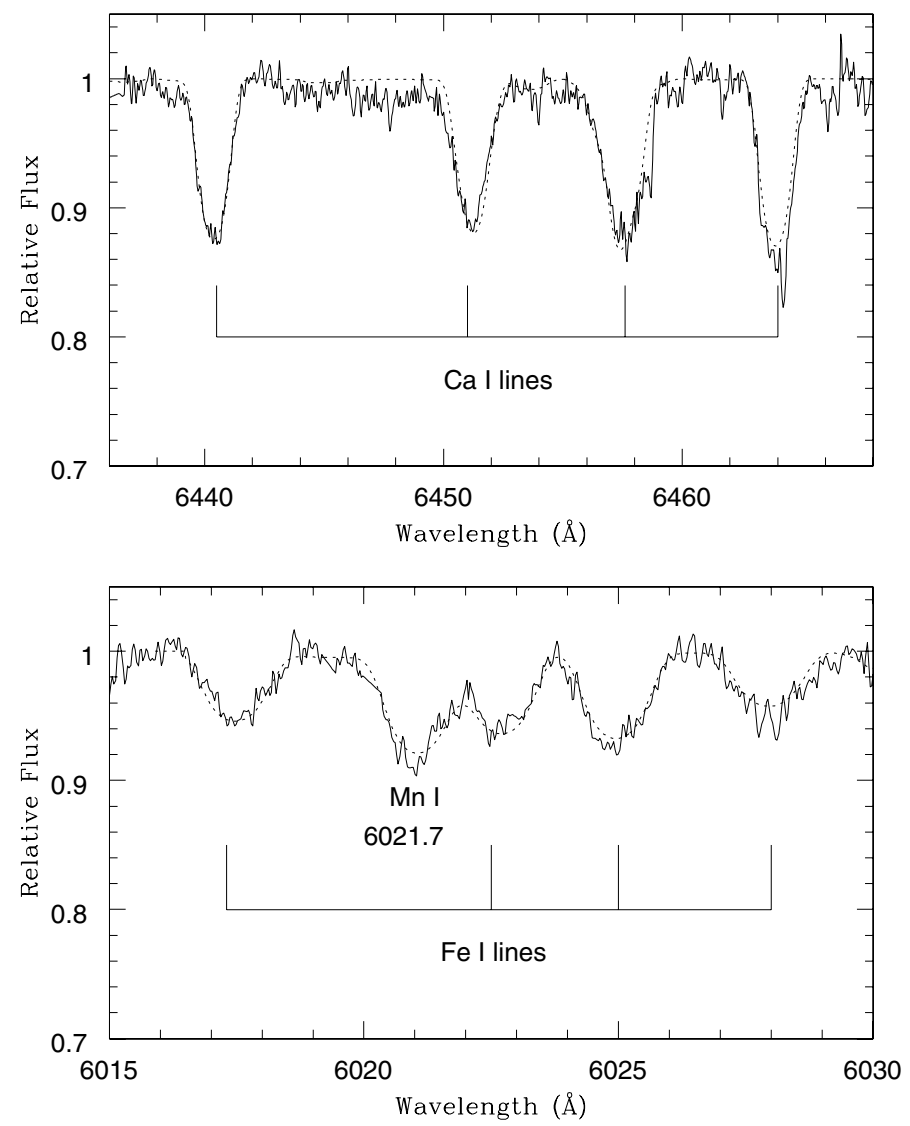

Fig. 4. Synthesis fit to the spectrum of IRAS $05381+1012$ in the regions 6436-6468 $\AA$ (top) and 6015-6030 ̊ (bottom). Full lines correspond to the observed spectrum and dotted lines are the synthetic spectrum calculated for $\log \epsilon(\mathrm{Ca})=5.96$ (top) and $\log \epsilon(\mathrm{Mn})=4.99$ (bottom), using $T_{\text {eff }}=5200 \mathrm{~K}$ and $\log g=1.0$.

within a 2- $\sigma$ uncertainty. The star is also slightly underabundant in the only s-process element detected (Barium).

\section{Discussion}

\subsection{Evolutionary status and distance}

With the derived stellar parameters of $T_{\text {eff }}=5200 \mathrm{~K}$ and $\log g=$ 1.0 , the post-AGB star IRAS $05381+1012$ can be located in the $\log g-\log T_{\text {eff }}$ plane and compared to the post-horizontal branch evolutionary tracks of Schönberner (1983) and Blöcker \& Schönberner (1990). These tracks are plotted in Fig. 5 for 
Table 2. Abundance results (in the scale $\log \epsilon(H)=12.0$ ).

\begin{tabular}{ccc}
\hline \hline Species & IRAS 0538+1012 & Sun $^{*}$ \\
\hline $\mathrm{Mg}$ I & $7.48 \pm 0.30$ & 7.58 \\
$\mathrm{Si} \mathrm{I}$ & $6.55 \pm 0.30$ & 7.55 \\
$\mathrm{Ca}$ I & $5.96 \pm 0.40$ & 6.36 \\
$\mathrm{Mn}$ I & $4.59 \pm 0.30$ & 5.39 \\
$\mathrm{Fe} \mathrm{I}$ & $6.72 \pm 0.20$ & 7.50 \\
$\mathrm{Ni}$ I & $5.75 \pm 0.30$ & 6.25 \\
$\mathrm{Ba}$ II & $1.93 \pm 0.20$ & 2.13 \\
\hline
\end{tabular}

* Anders \& Grevesse (1989).

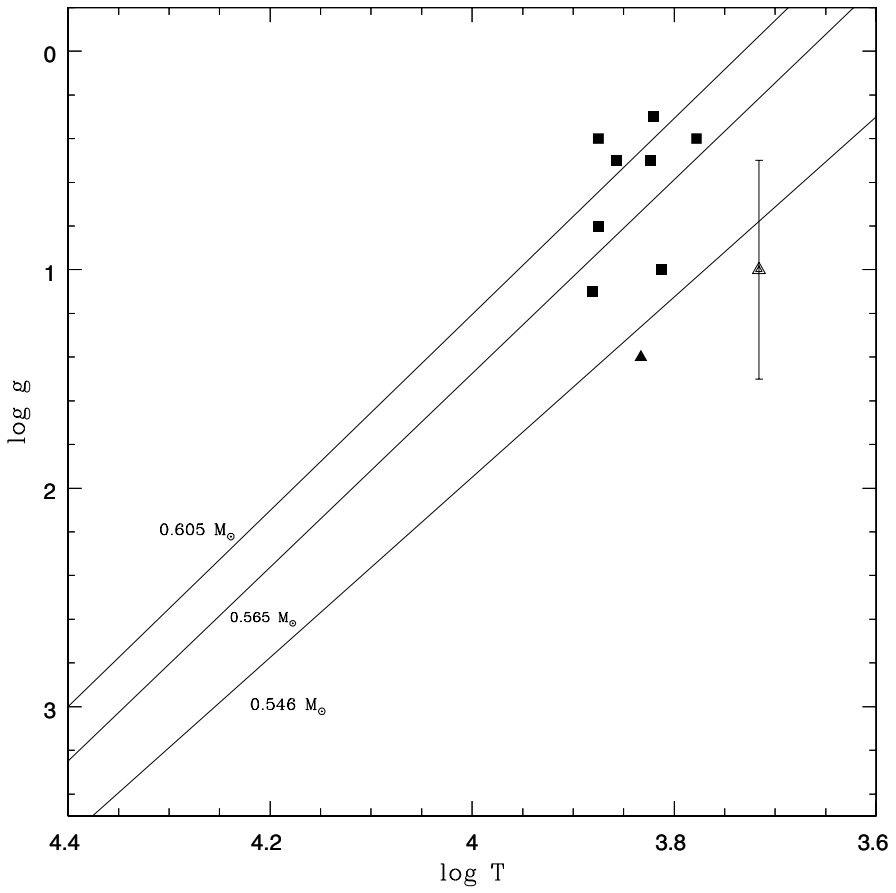

Fig. 5. Logarithmic surface gravity versus effective temperature diagram showing the location of IRAS $05381+1012$ (open triangle) according to the stellar parameters determined here. The diagram also shows the location of the post-AGB star IRAS 19386+0155 (filled triangle) taken from Pereira et al. (2004), and of other previously identified post-AGB objects (filled squares) taken from Table 1 of Luck (1993). Full lines indicate the post-HB tracks of Schönberner (1983) and Blöcker \& Schönberner (1990).

core masses of $0.546 M_{\odot}, 0.565 M_{\odot}$ and $0.605 M_{\odot}$, respectively. The positions of other previously studied post-AGB stars are also shown. The parameters suggest that the mass of IRAS $05381+1012$ is $M \simeq 0.55 M_{\odot}$ with a $2-\sigma$ uncertainty of 0.05 . This uncertainty is mainly related to the uncertainty in $\log g$, since $T_{\text {eff }}$ is much better constrained.

With the values of mass, gravity, and temperature in hand, we can estimate the distance $r$, in kpc, of IRAS $05381+1012$. The relation among these quantities with the $V$ magnitude and interstellar absorption $\left(A_{\mathrm{V}}\right)$ is given by:

$$
\begin{aligned}
\log r(\mathrm{kpc})= & \frac{1}{2}\left(\log \frac{M_{\star}}{M_{\odot}}+0.4\left(V-A_{\mathrm{V}}\right)\right. \\
& \left.+4 \log T_{\mathrm{eff}}-\log g-16.46\right) .
\end{aligned}
$$

Inserting the values $M_{\star}=0.55 M_{\odot}, T_{\text {eff }}=5200 \mathrm{~K}$, and $\log g=$ 1.0, this equation becomes:

$5 \log r(\mathrm{kpc})=\left(V-A_{\mathrm{V}}\right)-7.14$

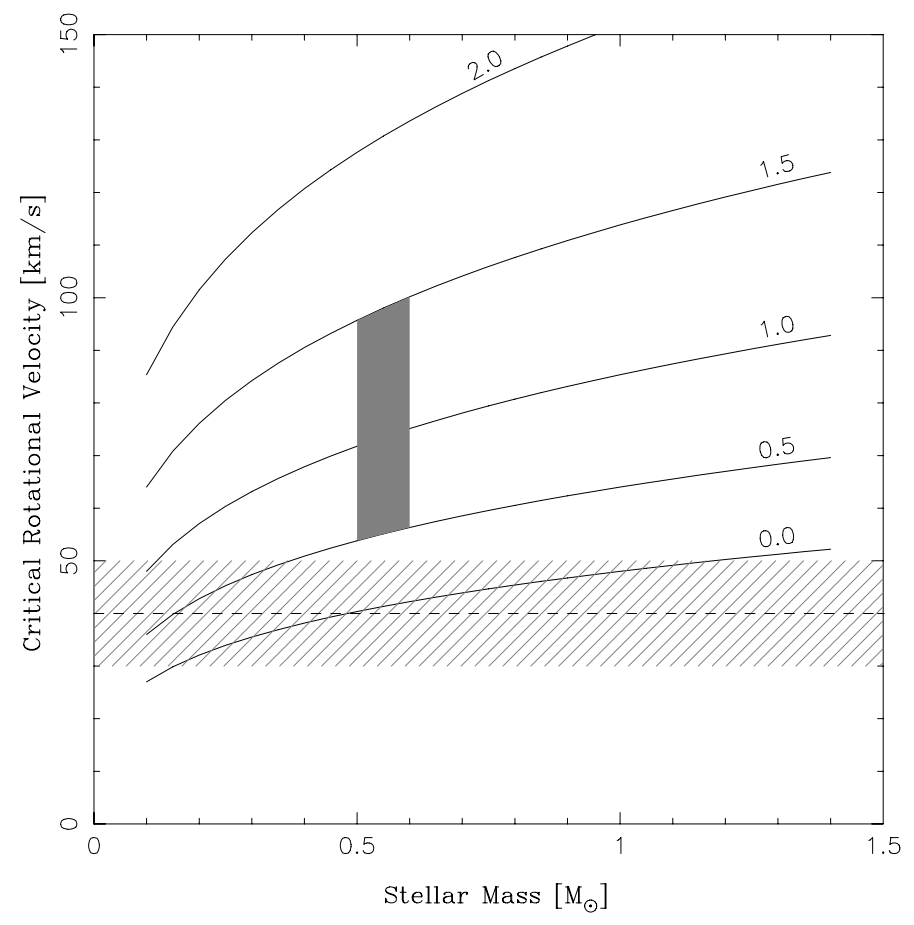

Fig. 6. Critical breakup velocity versus stellar mass for different values of $\log g$ from 0.0 to 2.0 (full lines). The figure shows the possible values of $v_{\text {crit }}$ for IRAS $05381+1012$ (gray square), and compares them to the value of $v \sin i$ (dashed line) and its corresponding uncertainty (hatched area).

which, for $V=10.6$ and $A_{\mathrm{V}}=1.3$ (Fujii et al. 2002), gives $r \simeq$ $2.7 \pm 0.5 \mathrm{kpc}$ and a luminosity of $\simeq 970 \pm 370 L_{\odot}$. The uncertainties in these quantities come mainly from the uncertainty in $\log g$. The errors in $M_{\star}$ and $T_{\text {eff }}$ do not contribute significantly.

\subsection{The rotational velocity}

The critical breakup velocity as a function of stellar mass and surface gravity can be written as:

$v_{\text {crit }}=\sqrt{\frac{2 G M_{\star}}{R_{\star}}}=48\left(g M_{\star}\right)^{1 / 4}$,

where $v_{\text {crit }}$ is in $\mathrm{km} \mathrm{s}^{-1}, M_{\star}$ is in units of $M_{\odot}$ and $g$ is in $\mathrm{cm} \mathrm{s}^{-2}$. Figure 6 shows the curves $v_{\text {crit }}$ versus $M_{\star}$ for several constant values of $\log g$ (full lines). The gray region corresponds to the possible values that $v_{\text {crit }}$ can take for IRAS $05381+1012$, according to the uncertainties in the stellar parameters. The dashed horizontal line corresponds to the estimated projected rotational velocity, and its corresponding uncertainty is represented by the hatched area. This figure clearly shows that the parameters estimated for IRAS $05381+1012$ are self consistent, in that its rotational velocity never exceeds the critical velocity, even in the most pessimistic cases. The estimated critical velocity for IRAS $05381+1012$ is $\sim 73 \mathrm{~km} \mathrm{~s}^{-1}$.

\subsection{The abundance pattern}

In this section, we compare the abundance pattern of IRAS $05381+1012$ to that of the population I supergiants. We choose these objects because the stellar parameters of IRAS $05381+1012$ indicate that its colder component would be a supergiant. Moreover, its position in the $\log g-\log T_{\text {eff }}$ plane 
Table 3. Metallicities and mean abundances of the $\langle[\alpha / \mathrm{H}]\rangle$ and $\langle[\mathrm{s} / \mathrm{H}]\rangle$ elements for population I supergiants and post-AGB stars.

\begin{tabular}{|c|c|c|c|c|}
\hline Class & $\langle[\mathrm{Fe} / \mathrm{H}]\rangle$ & $\langle[\alpha / \mathrm{H}]\rangle$ & $\langle[\mathrm{s} / \mathrm{H}]\rangle$ & Ref. \\
\hline $\begin{array}{l}\text { population I } \\
\text { supergiants }\end{array}$ & +0.13 & +0.23 & +0.18 & 1 \\
\hline $\begin{array}{l}21 \mu \mathrm{m} \\
\text { post-AGB }\end{array}$ & -0.3 to -1.0 & -0.37 & +0.78 & 2 \\
\hline $\begin{array}{l}\text { Metal-poor, } \\
\text { binary } \\
\text { post-AGB }\end{array}$ & -1.5 to -4.5 & -2.11 & - & 3 \\
\hline Star & {$[\mathrm{Fe} / \mathrm{H}]$} & $\langle[\alpha / \mathrm{H}]\rangle$ & $\langle[\mathrm{s} / \mathrm{H}]\rangle$ & \\
\hline HD 161796 & -0.3 & +0.1 & 0.0 & 4 \\
\hline HR 6144 & -0.4 & +0.1 & -0.2 & 4 \\
\hline HR 7671 & -1.1 & -0.7 & -0.5 & 4 \\
\hline 89 Her & -0.4 & -0.2 & 0.0 & 4 \\
\hline SAO 173329 & -0.8 & -0.7 & - & 5 \\
\hline HD 95767 & +0.1 & +0.1 & - & 5 \\
\hline SAO 239853 & -0.8 & -0.4 & -1.2 & 5 \\
\hline HD 107369 & -1.1 & -0.9 & -1.2 & 5 \\
\hline HD 108015 & -0.1 & 0.0 & - & 5 \\
\hline HD 131356 & -0.6 & -0.6 & - & 5 \\
\hline HD 133656 & -0.7 & -0.5 & -1.1 & 5 \\
\hline HR 4912 & -1.2 & -0.9 & -1.5 & 6 \\
\hline HD 172481 & -0.6 & -0.6 & -0.4 & 7 \\
\hline Hen 3-1312 & -1.1 & -0.6 & -0.3 & 8 \\
\hline IRAS & & & & \\
\hline $19386+0155$ & -1.1 & -0.3 & -1.1 & 9 \\
\hline IRAS & & & & \\
\hline $05381+1012$ & -0.8 & -0.5 & -0.2 & 10 \\
\hline
\end{tabular}

Refs.: 1: Luck \& Bond (1989); Luck \& Lambert (1985). 2: van Winckel \& Reyniers (2000). 3: Trams et al. (1993). 4: Luck et al. (1990). 5: van Winckel (1997). 6: Lambert et al. (1983). 7: Reyniers \& van Winckel (2001). 8: Pereira (2004). 9: Pereira et al. (2004). 10: This work.

coincides with other post-AGB stars that display similar atmospheric parameters to those of Population I supergiants.

As already mentioned, the high, projected rotational velocity of IRAS $05381+1012$ is a limiting factor in determining the abundances, especially for the weak lines. For example, some lines, like the carbon lines around 7110-7120 $\AA$ and the nitrogen lines around $8680-8685 \AA$, are washed away due to the rapid rotation of the star. Therefore, we are unable to obtain the abundance of some light elements, such as carbon, nitrogen, and oxygen, that are very useful for detecting traces of internal nucleosynthesis (like nitrogen enrichment due to CNO-cycle and carbon enrichment due to thermal pulses). We are rather limited to discussing the metallicity, the abundance of some $\alpha$-elements, and the abundance of only one element created by neutron capture nucleosynthesis.

Table 3 gives the metallicity, the mean abundance of the $\alpha$-elements, and the mean abundance of the elements created by slow neutron-capture nucleosynthesis $(\langle[\mathrm{s} / \mathrm{H}]\rangle)$. This table is divided in two parts. The top part gives the mean abundances and metallicity of population I supergiants (Luck \& Bond 1989; Luck \& Lambert 1985), of post-AGB stars that show the $21 \mu \mathrm{m}$ feature and that are also carbon and s-process enriched (Hrivnak 1995; van Winckel \& Reyniers 2000), and of metal-poor binary post-AGB stars (Trams et al. 1993). The bottom part gives the mean abundances and metallicities of post-AGB stars that do not belong to any of the classes mentioned above, individually named.

Due to their highest $\langle[\mathrm{s} / \mathrm{H}]\rangle$, the stars that show the $21 \mu \mathrm{m}$ feature are clearly overabundant, with respect to the Sun, in elements created by s-process nucleosynthesis. On the other hand, the mean $\langle[\mathrm{s} / \mathrm{H}]\rangle$ of population I supergiants is solar. The metallicity and the mean abundances of $\alpha$-elements for metalpoor, binary post-AGB star reflects that depletion due to gas-dust separation has taken place (Trams et al. 1993). The mean abundance of $\alpha$-elements for population I supergiants is solar, while for the stars showing the $21 \mu \mathrm{m}$ feature, the mean abundances reflect the chemical history of the Galaxy.

Comparing the mean abundance of IRAS $05381+1012$ with the mean abundance of the stars in the bottom part of Table 3, we see that there are at least three post-AGB stars that display similar mean abundances: Hen 3-1312, HR 7671, and HD 172481. It is normally assumed that post-AGB stars should show s-process enhancements, although this is not an absolute requirement. Actually, these overabundances have been found only in objects with clear carbon rich circumstellar material. Post-AGB stars that do not show s-process enhancements are more difficult to interpret in the framework of the standard AGB theory. The amount of s-process material depends on the number of thermal pulses when the star is at the AGB phase. Therefore, these stars are probably low-mass stars in which the third dredge-up would occur above a given mass limit (van Winckel 2003). This would explain why we have several post-AGB stars listed in Table 3 with mean abundances $\langle[\mathrm{s} / \mathrm{H}]\rangle$ below solar.

\section{Conclusions}

After analyzing the high-resolution optical absorption spectrum of IRAS $05381+1012$, we presented evidence that it is broadened due a rapid rotation of at least $40 \pm 10 \mathrm{~km} \mathrm{~s}^{-1}$.

In addition, we showed that IRAS $05381+1012$ present several spectral characteristics that have already been detected in the several post-AGB stars so far observed, including their high luminosity and lower gravity. Its position in the $\log g-$ $\log T_{\text {eff }}$ plane agrees with previous studies done for other post-AGB stars. In the HR-diagram, post-AGB stars occupy a narrow region and could be used as another constraint for extragalactic distances, due to its narrow luminosity function (Bond 1997). With our derived luminosity, the position of IRAS $05381+1012$ in the HR-diagram falls well inside a box where most post-AGB stars are located (Bond 1997).

Due to its abundance pattern, it is clear that IRAS $05351+1012$ belongs neither to the population I supergiants nor to any of the two categories of post-AGB stars shown in Table 3, i.e., metal-poor, binary post-AGB stars, and and the so-called " $21 \mu \mathrm{m}$ post-AGB stars". Its evolutionary status would be better constrained if we were able to detect some lines of the CNO elements. Yet, among several post-AGB stars already studied, many of them do not show overabundances of s-process (van Winckel 1997; Luck et al. 1990; Pereira et al. 2004) and IRAS $05381+1012$ is one of them.

Rapid rotation among AGB stars is very rare, although there are exceptions. This is the case for V Hya (Barnbaum et al. 1995), a star with very low surface gravity $(\log g=$ 0.0, Denn et al. 1991) that rotates at a velocity close to critical $\left(\simeq 14 \mathrm{~km} \mathrm{~s}^{-1}\right)$. Different mechanisms have been invoked to explain its rapid rotation, like common envelope binary evolution (Barnbaum et al. 1995), or a unseen compact companion (Sahai et al. 2003; Hirano et al. 2004). In this latter mechanism, the star would develop an accretion disk and a jet would be driven by it. Another example is ZNG 1, a post-AGB star in the globular cluster M 5. Dixon et al. (2004) use ultraviolet spectroscopy to show that this star has a rotational velocity $v \sin i=170 \pm 20 \mathrm{~km} \mathrm{~s}^{-1}$. 
The authors discuss several possibilities to explain this rapid rotation, including tidal spin-up by a binary companion. Another possibility could be a low-mass star (or a planet) companion that spirals into the AGB progenitor. This model has been proposed to explain the rapid rotation of K648 in the M 15 cluster (Alves et al. 2000).

In the case of IRAS $05381+1012$, it is not clear which would be the mechanism responsible for its rapid rotation. Interferometric studies in the infrared, as proposed by Barnbaum et al. (1995), would be one of the possibilities for investigating whether a disk exists around V Hya, and this kind of study might be applied to IRAS $05381+1012$ too. Other observations, such as looking for jets using $\mathrm{H} \alpha$ imaging (Sahai et al. 2003) or CO mapping (Hirano et al. 2004), have been already done for V Hya and should also be considered for IRAS $05381+1012$.

\section{References}

Anders, E., \& Grevesse, N. 1989, Geochim. Cosmochim. Acta, 53, 197 Alves, D., Bond, H., \& Livio, M. 2000, ApJ, 120, 2044

Balick, B. 1987, AJ, 94, 671

Barnbaum, C., Morris, M., \& Kahane, C. 1995, ApJ, 450, 862

Bloecker, T., \& Schoenberner, D. 1990, A\&A, 240, L11

Bond, H. 1997, IAU Symp., 180

Booth, A. J., Shallis, M. J., \& Wells, M. 1983, MNRAS, 205, 191

Denn, G. R., Luck, R. E., \& Lambert, D. L. 1991, ApJ, 377, 657

Dixon, W. D., Brown, T. M., \& Landsman, W. B. 2004, ApJ, 600, L43

Fujii, T., Nakada, Y., \& Parthasarathy, M. 2002, A\&A, 385, 884

Garcia-Lario, P., Manchado, A., Pych, W., \& Pottasch, S. R. 1997, A\&AS, 126, 479

Hamuy, M., Philips, M. M., Maza, J., et al. 1994, AJ, 108, 2226

Herwig, F. 2004, in Asymmetrical Planetary Nebulae III, AGB evolution, ed. M. Meixner, J. H. Kastner, B. Balick, \& N. Soker, 387

Hirano, N., Shinnaga, H., Dinh-V-Trung, et al. 2004, ApJ, 616, L43

Hrivnak, B. J., Kwok, S., \& Geballe, T. R. 1994, ApJ, 420, 783
Jacoby, G. H., Hunter, D. A., \& Christian, C. A. 1984, ApJS, 56, 257 Kaufer, A., Stahl, O., Tubbesing, S., et al. 1999, The Mesenger, 95, 8 Kurucz, R., \& Bell, B. 1995, CD-ROM 23, Atomic Line Data (Cambridge: SAO) Kwok, S. 1982, ApJ, 258, 280

Kwok, S. 1993, ARA\&A, 31, 63

Lambert, D. L., Luck, R. E., \& Bond, H. E. 1983, PASP, 95, 413

Luck, R. E., \& Bond, H. 1985, ApJ, 292, 559

Luck, R. E., \& Bond, H. 1989, ApJS, 71, 559

Luck, R. E., Bond, H. E., Lambert, D. L., et al. 1990, 357, 188

Luck, R. E., \& Lambert, D. 1985, ApJ, 298, 782

Luck, R. E. 1993, in Luminous High-Latitude Stars, The chemical composition of luminous high-latitude stars post-AGB stars, ed. D. D. Sasselov, 87

Martin, G. A., Fuhr, J. R., \& Wiese, W. L. 1988, J. Phys. Chem. Ref. Data, 17, 4 McCausland, R. J. H., Conlon, E. S., Dufton, P. L., \& Keenan, F. P. 1992, ApJ, 394, 298

Meixner, M., Ueta, T., Dayal, A., et al. 1999, ApJS, 122, 221

Pereira, C. B., \& Machado, M. A. D. 2003, A\&A, 407, 311

Pereira, C. B., Lorenz-Martins, S., \& Machado, M. 2004, A\&A, 422, 637

Pereira, C. B. 2004, A\&A, 413, 1009

Pereira, C. B., Smith, V. V., \& Cunha, K. 2005, A\&A, 429, 993

Ryans, R. S. I., Dufton, P. L., Mooney, C. J., et al. 2003, A\&A, 401, 1119

Sahai, R., Morris, M., Knapp, G. R., Young, K., \& Barnbaum, C. 2003, Nature, 426, 261

Schmidt-Kaler, T. 1982, in Landolt-Börnstein New Series, ed. K. Schaifers, \& H. H. Vigt, Group 4, Vol. 2b (Berlin: Springer), 449

Smith, G., Edvardsson, B., \& Frisk, U. 1986, A\&A, 165, 126

Schoenberner, D. 1983, ApJ, 272, 708

Sneden, C. 1973, Ph.D. Thesis, Univ. of Texas

Smith, V. V., Pereira, C. B., \& Cunha, K. 2001, ApJ, 556, L55

Thévenin, F. 1990, A\&AS, 82, 179

Trams, N. R., Waelkens, C., \& Waters, L. B. F. M. 1993, in Luminous HighLatitude Stars, Extremely metal-poor post-AGB stars, ed. D. D. Sasselov, 103

van Winckel, H. 1997, A\&A, 319, 561

van Winckel, H. 2003, ARA\&A, 41, 391

van Winckel, H., \& Reyniers, M. 2000, A\&A, 345, 135

Waelkens, C., Van Winckel, H., Bogaert, E., \& Trams, N. R. 1991, A\&A, 251, 495

Wiese, W. L., Smith, M. W., \& Miles, B. M. 1969, NBS Ref. Data. Ser. 